\title{
Kahramanmaraş-Andırın Yöresi Relikt Doğu Kayını (Fagus orientalis Lipsky) Ormanlarında Kırmızı Renkli Yapışkan Tuzaklarla Tespit Edilen Kabuk ve Ambrosya Böcekleri (Col.: Curculionidae: Scolytinae ve Platypodinae) Üzerine Notlar
}

\author{
*Oğuzhan Sarıkaya \\ Isparta Uygulamalı Bilimler Üniversitesi, oguzhansarikaya07@gmail.com, \\ Araştırma Makalesi \\ Geliş Tarihi: 17.05.2018 \\ Kabul Tarihi: 05.11.2018
}

$\ddot{O} z$

Türkiye ormanlarının asli ağaç türlerinden olan, Doğu kayını (Fagus orientalis Lipsky), esas yayılışını kuzey bölgelerde yapmakla birlikte ülkemizin güney doğu bölümünde özellikle Adana, Osmaniye, Hatay ve Kahramanmaraş yörelerinde izole kalmış, relikt Doğu kayını sahaları bulunmaktadır. Bu çalışmada, Andırın (Kahramanmaraş) Doğu kayını ormanlarında yayılış gösteren kabuk ve ambrosya böcekleri tespit edilmiştir. Arazi çalışmaları Andırın-Akifiye yöresinde (37 $\left.44^{\prime} \mathrm{K}, 36^{\circ} 22^{\prime} \mathrm{D}\right) 2015$ ve 2016 yıllarında mart ayı başlarından itibaren ekim ayı sonuna kadar olan periyodu içerisine alan süreçte yürütülmüştür. Bu amaçla, saha geneline Kırmızı renkli yapışkan tuzaklar (\%96’l1k etil alkol ve \% 1 toluenden oluşan cezbedici karışım ile) tesis edilmiştir. Kontroller, ayda bir kez olacak şekilde gerçekleştirilmiş ve her kontrolde Kırmızı renkli yapışkan tuzaklar yenisiyle değiştirilmiştir. Elde edilen toplam 1245 adet bireyin incelenmesi sonucu Curculionidae familyasının Platypodinae alt familyasından 1 tür ve Scolytinae alt familyasının 3 farklı tribusuna bağlı 7 tür olmak üzere toplam 8 tür belirlenmiştir. Bu türler; Platypus cylindrus (Fabricius, 1792) (Col.: Curculionidae, Platypodinae), Taphrorychus ramicola (Reitter, 1894), T. villifrons (Dufour, 1843), Scolytus intricatus (Ratzeburg, 1837), Anisandrus dispar (Fabricius, 1792), Trypodendron signatum (Fabricius 1787), Xyleborinus saxesenii (Ratzeburg, 1837) ve Xyleborus monographus (Fabricius, 1792)'tur. Toplanan türler arasında Xyleborinus saxesenii en yoğun tür olarak tespit edilmiş, X. saxesenii'yi, sırasıyla Anisandrus dispar, Taphrorychus ramicola ve Trypodendron signatum takip etmiştir.

Anahtar kelimeler: Fagus orientalis, Kabuk ve ambrosya böcekleri, Kahramanmaraş-Andırın, Kırmızı kanatlı yapışkan tuzak

\section{Notes on Bark and Ambrosia Beetles (Col.: Curculionidae: Scolytinae and Platypodinae) Determined By Red Winged Sticky Traps in Relict Oriental Beech (Fagus orientalis Lipsky) Forests of Kahramanmaraş-Andırın Province}

*Oğuzhan Sarikaya

Isparta University of Applied Sciences, oguzhansarikaya07@gmail.com

\begin{abstract}
The main distribution area of oriental beech (Fagus orientalis Lipsky) which is one of main tree species for Turkish forests, is in the northern region of Turkey. By the way, isolated relict oriental beech forest exists in south-eastern Turkey especially Adana, Osmaniye, Hatay, and Kahramanmaraş.

In this study, bark and ambrosia beetle species which distribute on oriental beech forests of Andırın (Kahramanmaraş) were determined. The survey was carried out in Andirın-Akifiye province ( $\left.37^{\circ} 44^{\prime} \mathrm{N}, 36^{\circ} 22^{\prime} \mathrm{E}\right)$ in the south-eastern part of Turkey. Field studies were conducted from March to October in 2015 and 2016. For this aim, the red winged sticky traps (by mixed of ethyl alcohol $96 \%$ and toluen $1 \%$ ) were set in stand. Checking was made monthly and traps were replaced with new ones at each controlling period. Based on the material collected from Oriental Beech forests in Andırın between 2015 and 2016 by red winged
\end{abstract}

* Sorumlu Yazar: Isparta Uygulamalı Bilimler Üniversitesi, Orman Fakültesi, 32260, ISPARTA, oguzhansarikaya07@gmail.com 
sticky traps and by examination on 1224 individuals during 2 years, a total of 8 bark and ambrosia beetle species belonging to Platypodinae and 6 genera of 3 tribe from Scolytinae were identified. These species are; Platypus cylindrus (Fabricius, 1792) (Col.: Curculionidae, Platypodinae), Taphrorychus ramicola (Reitter, 1894), T. villifrons (Dufour, 1843), Scolytus intricatus (Ratzeburg, 1837), Anisandrus dispar (Fabricius, 1792), Trypodendron signatum (Fabricius 1787), Xyleborinus saxesenii (Ratzeburg, 1837) and Xyleborus monographus (Fabricius, 1792). Among those, X. saxesenii was the more abundant species than the others. The species of $A$. dispar, T. ramicola and $T$. signatum were followed it respectively.

Keywords: Fagus orientalis, ambrosia and bark beetles, Kahramanmaraş-Andırın, Red winged sticky trap

\section{GíRiș}

Ormanlar ekolojik fonksiyonlarını, dünya üzerinde birçok önemli habitat tipini oluşturarak ve içerisinde barındırdığı biyolojik çeşitlilik ile yerine getirmektedir [1]. Ayrıca ormanlar, orman içi mera ve su ekosistemleri, odun ve odun dışı ürün ve hizmetleri ile sayısız ölçülen ve ölçülemeyen fayda ve fonksiyonlara sahiptir. Ekosistem bitki, toprak ve su kaynakları dengesinin, kırsal alandaki sosyal istikrarın, barajların uzun ömürlü olmasının ve gıda güvenliğinin temel sigortasıdır [2]. Orman ekosistemleri, sürdürülebilir kalkınma sürecinin odak noktasında bulunmaktadırlar. Bu sürecin temel öğesi orman olup, başta odun hammaddesi olmak üzere diğer bütün üretimler ve faydalar buna bağımlıdır. Sistemde; toprak, meralar, orman içi sular, rekreasyon alanları, yaban hayvanları, bitkiler, yeraltı madenleri vb. diğer öğeler de yer almaktadır.

Orman ağaçları genellikle, doğal seleksiyon sonucunda bulundukları yöreye en iyi uyum sağlayabilen ve o yörede en üstün yaşama özelliklerini gösteren bireylerdir [3].

Ülkemizdeki kayın türleri bulundukları yapraklı ormanlarda baskın tür olarak görülmektedir. Nitekim, ülkemiz ormanlarının 1.621.256,6 hektarı normal, 340.402,9 hektarı ise bozuk olmak üzere toplam 1.961.659,5 hektarını kayın ormanı oluşturmaktadır [4].

Ülkemizde yayılış gösteren türlerden Avrupa kayını (Fagus sylvatica L.) ve Doğu kayını (Fagus orientalis Lipsky) birbirlerine çok benzemektedirler. Her ikisinde de gri renkli pürüzsüz bir gövde bulunurken, Doğu kayınının gövdesi biraz daha açık renktedir. Doğu kayınının yaprakları yumurta şeklinde ve lobsuz olup, Avrupa kayını ile Doğu kayını arasındaki en önemli fark kupula üzerindeki pullardır $[5,6]$.

Anavatanı ülkemiz olan ve doğal yayılışının büyük bir kısmını ülkemizde gerçekleştiren doğu kayını (Fagus orientalis) dünya üzerinde Bulgaristan'dan başlayarak, Türkiye, Kafkasya ve İran'da doğal olarak yayılış göstermektedir. Ülkemizdeki en geniş yayılışını Demirköy'den Hopa'ya kadar Karadeniz sahiline paralel uzanan dağların orta ve yüksek kısımlarında ve özellikle kuzey bakılarda kurduğu saf ve karışık ormanlarda yapmaktadır. Doğu Akdeniz'de Adana'nın Pos ormanlarında, Amanos Dağları'nda, Yozgat-Çekerek’te, Kahramanmaraş-Andırın yöresi ile Kütahya çevresinde ise lokal ve relikt olarak bulunmaktadır. Doğu kayını rutubetçe zengin, yağışın dengeli olduğu 1lıman iklimlerden hoşlanır $[7,8]$.

Ülkemizde, kuzey bölgelerdeki yayılışının (150-200 m) aksine kayın sahaları güney bölgelerde 1000-2000 m arasında yer alan yüksek rakımlarda bulunmaktadır. Doğu Akdeniz'de yer alan Doğu kayını sahaları, kayının esas yayılış alanından uzak mesafede konumlanmış olup, relikt ormanlar ve izole popülasyonlar olarak karakterize edilmektedir. Bu sahalar önemli gen kaynaklarıdır [9].

Türkiye ormanlarının sağlığını etkileyen çeşitli faktörler arasında böceklerin en başta geldiği kabul edilmektedir. Ülkemizde uygun iklim koşullarının ve dolayısıyla çok çeşitli bitki türlerinin bulunması, ormanlarda yıllarca varlığını hissettiren birçok böceğin yaşamlarını elverişli kılmıştır. Bugüne kadar Doğu Akdeniz'de yayılış gösteren ormanlarda zararlı böcek türleri ile ilgili çalışmalar olmakla birlikte Doğu kayını sahalarında çalışmalar yeterli değildir.

Scolytinae altfamilyası, kabukta üreyen kabuk böcekleri ve odunda üreyen kabuk böcekleri (ambrosya böcekleri) olmak üzere başlıca iki grupta incelenmektedir [10]. Kabukta üreyen kabuk böcekleri besince zengin floem ile kabuk içinde beslenirken, Ambrosya böcekleri galerilerini fizyolojik olarak zayıflayan bitkilerin özsuyu akışı yüksek odun dokusu içinde açarak simbiyotik fungusu bulaştırır ve üzerinde beslenmektedirler [11].

Bu çalışmada, Kahramanmaraş-Andırın yöresinde relikt Doğu kayını sahalarında yayılış gösteren kabuk ve ambrosya böcek türleri (Coleoptera: Curculionidae: Scolytinae) tespit edilmiştir

\section{MATERYAL VE METOD}

Kahramanmaraş - Andırın yöresinde yayılış gösteren relikt Doğu kayını sahalarında kabuk ve ambrosya böceklerini tespit etmek amacıyla arazi çalışmaları Akifiye mevkiinde (37 $\left.44^{\prime} \mathrm{K}, 36^{\circ} 22^{\prime} \mathrm{D}\right)$ (Şekil 1) 2015 ve 2016 yıllarında mart ayı başlarından itibaren ekim ayı sonuna kadar olan periyodu içerisine alan süreçte yürütülmüştür. 


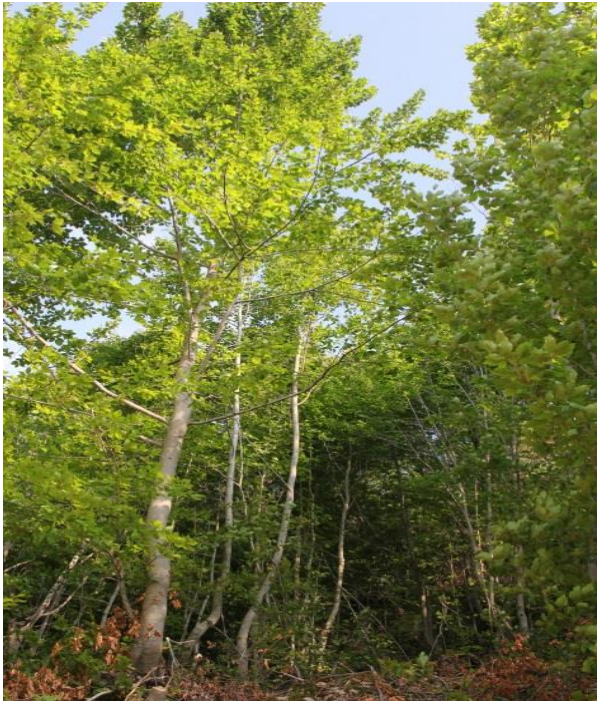

Şekil 1. Akifiye Doğu kayını sahası

Saha genelinde kabuk ve ambrosya türlerinin tespiti amacıyla saha geneline Kırmızı renkli yapışkan Rebell Rosso tuzaklar (\%96'l1k etil alkol ve \% 1 toluenden oluşan cezbedici karışım ile) tesis edilmiştir. Tuzaklar kırmızı renkte, iki adet $15 \times 21 \mathrm{~cm}$ ebatlarında ve üzeri yapışkan madde ile kaplı plastik levhaların birbirine geçirilmesiyle oluşmaktadır (Şekil 2).

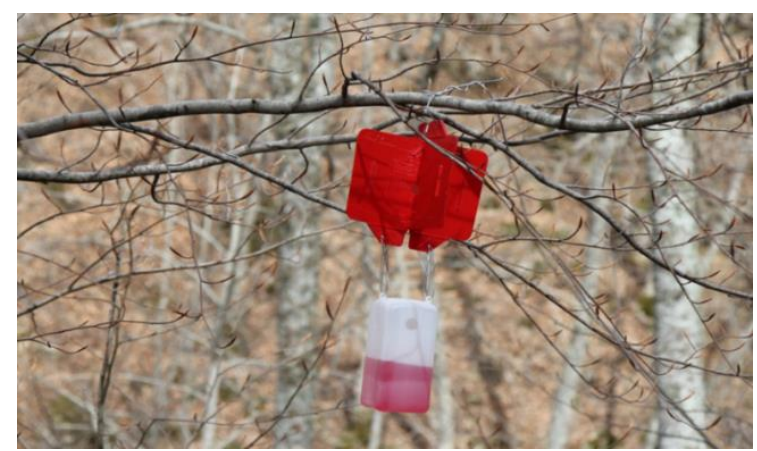

Şekil 2. Kırmızı kanatlı yapışkan tuzak (Rebell Rosso)
Tuzaklar ağaçların dallarına, birbirine en az 80-100 m aralıklarla yerden 2-2.5 m yüksekliğe asılmıştır. Kontroller, ayda bir kez olacak şekilde gerçekleştirilmiş ve her kontrolde Kırmızı renkli yapışkan tuzaklar yenisiyle değiştirilmiştir. Arazide tuzak kontrollerine ergin yakalaması bitinceye kadar devam edilmiştir.

\section{BULGULAR VE TARTIŞMA}

Kahramanmaraş-Andırın yöresinde relikt Doğu kayını (Fagus orientalis) sahalarında 2015 ve 2016 yıllarında kırmızı yapışkan tuzaklar kullanılarak yürütülen çalışmalarda, kabuk ve ambrosya böceklerinden toplam 8 tür belirlenmiştir.

Elde edilen toplam 1245 adet bireyin incelenmesi sonucu Curculionidae familyasının Platypodinae alt familyasından 1tür ve Scolytinae alt familyasının 3 farklı tribusuna bağlı 7 tür olmak üzere toplam 8 tür elde edilmiştir. Türlerin, alt famiya, tribus ve cinslerin sıralamasında Faunaeur [12] ve Knížek [13]'den faydalanılmıştır. Elde edilen türler Tablo 1 'de verilmiştir.

Akifiye Doğu kayını ormanlarında Kırmızı renkli yapışkan Rebell Rosso tuzaklar yardımıyla gerçekleştirilen çalışmalarda, 2015 yılında 591 birey, 2016 yılında ise 654 birey olmak üzere toplam 1245 adet birey elde edilmiştir. Toplanan türler arasında Xyleborinus saxesenii (\%70.12) en yoğun tür olarak tespit edilmiş, $X$. saxesenii'yi, sırasıyla Anisandrus dispar (\%14.62), Taphrorychus ramicola (\%3.86) ve Trypodendron signatum (\%3.69) takip etmiştir.

Tuzaklarda yapılan kontrollerde 2015 yılında 7 tür tespit edilirken, 2016 yılında 8 tür elde edilmiştir. Türlerden, Scolytus intricatus yalnızca 2016 y1lında tespit edilebilmiştir.

Kahramanmaraş-Andırın yöresi Doğu kayını sahalarında tespit edilen kabuk ve Ambrosya böceklerinin ülkemizdeki yayılış ve konukçularına ilişkin daha önce gerçekleştirilen çalışmalardaki kayıtlar Tablo 2'de verilmiştir. 
Tablo 1. Kahramanmaraş-Andırın Doğu kayını sahalarında Kırmızı yapışkan tuzaklar yardımıyla tespit edilen Kabuk ve Ambrosya böcekleri ve yıllara göre elde edilen birey sayıları

\begin{tabular}{|c|c|c|c|c|c|c|}
\hline \multirow[t]{2}{*}{ Alt Familya } & \multirow[t]{2}{*}{ Tribus } & \multirow[t]{2}{*}{ Tür } & \multicolumn{3}{|c|}{ Birey sayıları } & \multirow{2}{*}{$\begin{array}{c}\begin{array}{c}\text { Bulunma } \\
\text { Oranı }\end{array} \\
\%\end{array}$} \\
\hline & & & 2015 & 2016 & $\mathrm{~N}$ & \\
\hline \multicolumn{7}{|l|}{ Platypodinae } \\
\hline \multicolumn{7}{|c|}{ Platypodini } \\
\hline & & Platypus cylindrus (Fabricius, 1792) & 12 & 9 & 21 & 1.69 \\
\hline \multicolumn{7}{|l|}{ Scolytinae } \\
\hline \multicolumn{7}{|c|}{ Dryocoetini } \\
\hline & & Taphrorychus ramicola (Reitter, 1894) & 21 & 27 & 48 & 3.86 \\
\hline & & Taphrorychus villifrons (Dufour, 1843) & 12 & 17 & 29 & 2.33 \\
\hline \multicolumn{7}{|c|}{ Scolytini } \\
\hline & & Scolytus intricatus (Ratzeburg, 1837) & - & 13 & 13 & 1.04 \\
\hline \multicolumn{7}{|c|}{ Xyleborini } \\
\hline & & Anisandrus dispar (Fabricius, 1792) & 98 & 84 & 182 & 14.62 \\
\hline & & Xyleborus monographus (Fabricius, 1792) & 18 & 15 & 33 & 2.65 \\
\hline & & Trypodendron signatum (Fabricius 1787) & 21 & 25 & 46 & 3.69 \\
\hline & & Xyleborinus saxesenii ( Ratzeburg, 1837) & 409 & 464 & 873 & 70.12 \\
\hline & & Toplam & 591 & 654 & 1245 & 100 \\
\hline
\end{tabular}

Tablo 2. Elde edilen türlerin Türkiye'deki konukçu ve yayılışına ilişkin kayıtlar

\begin{tabular}{|c|c|c|}
\hline Türler & \multicolumn{2}{|c|}{ Türlere İlişkin Kayıtlar } \\
\hline & Konukçu Ağaç Türleri & Yayılışa İlişkin Kayıtlar \\
\hline \multicolumn{3}{|l|}{ Platypodinae } \\
\hline Platypus cylindrus & $\begin{array}{l}\text { Fagus orientalis, Fraxinus ornus, Prunus } \\
\text { avium, Quercus cerris, } Q . \text { frainetto, } \\
\text { Q.robur }\end{array}$ & $\begin{array}{l}\text { Bursa, Düzce, Hatay, İstanbul, } \\
\text { Sakarya }\end{array}$ \\
\hline \multicolumn{3}{|l|}{ Scolytinae } \\
\hline Taphrorychus ramicola & $\begin{array}{l}\text { Carpinus orientalis, Corylus avellana, } \\
\text { Fagus orientalis, Quercus cerris }\end{array}$ & $\begin{array}{l}\text { Bartın, Hatay, Isparta, Sakarya, } \\
\text { Trabzon }\end{array}$ \\
\hline Taphrorychus villifrons & $\begin{array}{l}\text { Carpinus betulus, Fagus orientalis, } \\
\text { Quercus cerris, } Q \text {. frainetto, Liquidambar } \\
\text { orientalis }\end{array}$ & $\begin{array}{l}\text { Amasya, Ankara, Bolu, Bursa, Hatay, } \\
\text { Isparta, İstanbul, Karabük, Sakarya, } \\
\text { Sinop, Tokat }\end{array}$ \\
\hline Scolytus intricatus & $\begin{array}{l}\text { Fagus orientalis, Ostrya carpinifolia, } \\
\text { Quercus cerris, } Q . \text { frainetto, } Q . \text { petraea, } \\
Q . \text { robur }\end{array}$ & Düzce, Hatay, Isparta, İstanbul, Sinop \\
\hline Anisandrus dispar & $\begin{array}{l}\text { Actinidia chinensis, Carpinus betulus, } \\
\text { Castanea sativa, Corylus avellana, Malus }\end{array}$ & $\begin{array}{l}\text { Adana, Ankara, Artvin, Bartın, Bolu, } \\
\text { Bursa, Çorum, Denizli, Giresun, } \\
\text { Gümüşhane, Hatay, Isparta, İstanbul, }\end{array}$ \\
\hline
\end{tabular}




\begin{tabular}{|c|c|c|}
\hline & $\begin{array}{l}\text { domestica, Populus nigra, } \quad \text { Prunus } \\
\text { cerasus, Quercus sp., Tilia sp., Ulmus sp. }\end{array}$ & $\begin{array}{l}\text { Karabük, Kastamonu, Muğla, Niğde, } \\
\text { Ordu, Rize, Sakarya, Samsun, } \\
\text { Trabzon, Zonguldak }\end{array}$ \\
\hline Xyleborus monographus & $\begin{array}{l}\text { Castanaea sativa, Fagus orientalis, } \\
\text { Quercus frainetto }\end{array}$ & Bursa, Hatay, İstanbul, Kocaeli \\
\hline Trypodendron signatum & Quercus sp., Fagus orientalis, Alnus sp. & $\begin{array}{l}\text { Bolu, Gümüşhane, Isparta, İstanbul, } \\
\text { Karabük, Sakarya, Sinop, Trabzon }\end{array}$ \\
\hline Xyleborinus saxesenii & $\begin{array}{l}\text { Abies cilicica, A. nordmanniana subsp. } \\
\text { bornmuelleriana, Actinidia chinensis, } \\
\text { Alnus sp., Cedrus libani, Corylus avellana, } \\
\text { Fagus orientalis, Ficus carica, Fraxinus } \\
\text { ornus, Juglans regia, Juniperus excelsa, } \\
\text { Liquidambar orientalis, Pinus nigra, } \\
\text { Prunus armeniaca, P. avium, Pyrus } \\
\text { communis, Quercus cerris }\end{array}$ & $\begin{array}{l}\text { Amasya, Antalya, Artvin, Bolu, } \\
\text { Düzce, Giresun, Hatay, Isparta, } \\
\text { İstanbul, Kocaeli, Konya, Mersin, } \\
\text { Muğla, Ordu, Rize, Sakarya, Samsun, } \\
\text { Sinop, Trabzon, Zonguldak }\end{array}$ \\
\hline
\end{tabular}

* Türlerin Türkiye'deki konukçu ve yayılış bilgilerine ilişkin kayıtlar şöyledir: [10, 14-27]

\section{SONUÇLAR VE ÖNERİLER}

Kahramanmaraş-Andırın yöresinde relikt Doğu kayını sahalarında kırmızı renkli yapışkan tuzaklar ile yürütülen arazi çalışmalarında, toplam 8 tür tespit edilmiş olup, belirlenen Kabuk ve Ambrosya türlerinin tamamı Kahramanmaraş yöresi için yeni kayıt niteliğindedir. Tespit edilen türlerden Anisandrus dispar ülkemizde Doğu Kayını (Fagus orientalis) üzerinde ilk kez tespit edilmektedir.

Türlerden, Taphrorychus ramicola, Taphrorychus villifrons, Xyleborus monographus ve Scolytus intricatus'un Doğu Akdeniz Bölgesinde Hatay-Hassa'daki Doğu kayını sahalarında daha önce yapılan çalışmalarda tespit edildiği bildirilmektedir [18,28,29]. Ancak, Anisandrus dispar, Trypodendron signatum ve Xyleborinus saxesenii'nin Doğu Akdeniz Bölgesi'ndeki relikt Doğu kayını sahalarında ilk kez bu çalışmayla tespit edildiği görülmektedir.

Doğu Akdeniz'de yer alan, relikt ormanlar ve izole popülasyonlar olarak görülen ve bu yönüyle önemli gen kaynakları olarak tasvir edilen bu sahalarda Kabuk ve Ambrosya böcekleri ile ilgili daha detaylı çalışmaların yürütülmesinin gerekliliği görülmektedir.

\section{TEŞEKKÜR}

Veri, bilgi ve yöntemler, UNDP Türkiye tarafından Orman Genel Müdürlüğü işbirliğinde yürütülen GEF destekli "Entegre Orman Yönetimi” projesi kapsamında üretilmiştir. $\mathrm{Bu}$ kapsamda, UNDP Türkiye ve Orman Genel Müdürlüğüne teşekkür ederim.

\section{KAYNAKLAR}

[1] W.D. Pearce and G.T.C. Pearce, The Value of Foret Ecosystems. A Report to The Secretariat Convention on Biological Diversity (2001).

[2] M. Konukçu, Ormanlar Ve Ormancılığımız: Faydaları, İstatistiki Gerçekler, Anayasa, Kalkınma Planları, Hükümet Programları ve Y1llık Programlarda Ormancilık. Devlet Planlama Teşkilatı Yayınları, Ankara, (2001)
[3] K. Işık, Biyolojik Çeşitlilik ve Orman Gen Kaynaklarımız. Orman Bakanlığı Yayın No. 13, Ankara, (1996).

[4] Anonim, Orman Atlası. Orman Genel Müdürlüğü Yayınları, Ankara, (2013).

[5] Anonim, Kayın. Ormancılık Araştırma Enstitüsü Yayınları, Ankara, (1985).

[6] G. Kandemir and Z. Kaya, Fagus orientalis, Lipsky. Technical guidelines of EUFORGEN, (2009).

[7] R. Anşin and Z.C. Özkan, Tohumlu Bitkiler. Odunsu Taksonlar. KTÜ Orman Fakültesi Yayınları, Trabzon, (1997)

[8] İ. Atalay, Kayın Ormanlarının Ekolojisi Ve Tohum Transfer Yönünden Bölgelere Ayrılması. Orman Ağaçları ve Tohumları Islah Araştırma Müdürlüğü yayınları, Ankara, (1992).

[9] M. Y1lmaz, Is there a Future for the Isolated Oriental Beech (Fagus orientalis Lipsky) Forests in Southern Turkey. Acta Silvatica \& Lignaria Hungarica 6 : 111-114, (2010).

[10] E. Selmi, Türkiye Kabuk Böcekleri ve Savaşı. İstanbul Üniversitesi Yayınları, İstanbul, (1998).

[11] R. Kushiyev, O. Aker, and C. Tuncer, Ambrosya Böcekleri (Coleoptera: Curculionidae: Scolytinae ve Platypodinae) ile Ambrosya Fungusları Arasındaki Simbiyotik İlişkiler. Harran Tarım ve Gıda Bilimleri Dergisi 21(2): 239-246, (2017).

[12] Fauna Europaea, https://fauna-eu.org/, (accessed on: 05.01.2017).

[13] M. Knížek, Scolytinae, In: Löbl, I. ve Smetana, A. (Eds), Catalogue of Palaearctic Coleoptera, Vol. 7., Apollo Books, Stenstrup, 86-87, 204-251, (2011).

[14] E. Schimitschek, Fortinsekten der Turkei und ihre Umwelt. Grundlagen der Türkischen Forstentomologie, Prag, (1944).

[15] B. Alkan, Kizılcahamam, Bolu (Abant) ve Düzce ormanlarında yapılan Entomolojik Araştırmalar. Orman ve Av 3: 112-119, (1946)

[16] B. Alkan, Fındık Ağaçlarının Zararlıları ve Korunma Çareleri. Türk Yüksek Ziraat Mühendisler Birliği Yayınları, Ankara, (1948). 
[17] B. Alkan, Türkiye'nin bitki zararlısı kabuk böcekleri (Col., Scolytinae) faunası üzerine çalışmalar. Ankara Üniversitesi Ziraat Fakültesi Y1llığı. 14: 345-401, (1964).

[18] K.E. Schedl, Borkenkäfer aus der Türkei, II. Mitteilung 190. Beitrag zur Morphologie and Systematik der Scolytoidea. 34: 184-188, (1961).

[19] C. Chararas, Comportement de Xyleborus saxeseni Ratz., a l'égard de Liquidambar orientalis Mill., essence typique de l'Asie Mineure (Turquie). Comptes rendus de l'Académie des Sciences 260: 2313-2315, (1965).

[20] M. Kaya, Studies on Population Fluctuations of Adults of Xyleborus dispar (F.) (Coleoptera: Scolytidae) on Different Fruits in Bursa Province. Yüzüncü Yıl University, Agriculture Faculty, Journal of Agriculture Sciences 14: 113-117, (2004).

[21] H.H. Cebeci and H. Ayberk, Ambrosia beetles, hosts and distribution in Turkey with a study on the species of Istanbul province. African Journal of Agricultural Entomology 5: 1055-1059, (2010).

[22] E. Selmi, Scolytinae of Turkey. http://www.orman.istanbul.edu.tr/content/Scolytinae-

turkey), (accessed on: 19.01.2013).

[23] İ. Saruhan and H. Akyol, Monitoring population density and fluctuations of Anisandrus dispar and Xyleborinus saxesenii (Coleoptera: Scolytinae, Curculionidae) in hazelnut orchards. African Journal of Biotechnology 11:
4202-4207, (2012).

[24] Y. Yıldız, Bartın Ve Karabük Ormanlarının Scolytidae Faunası ve Bazı Önemli Türlerin Biyolojilerinin Belirlenmesi. Doktora Tezi. Bartın Üniversitesi, Fen Bilimleri Enstitüsü, Orman Mühendisliği Anabilim Dalı, Bartın, (2012).

[25] O. Sarıkaya, Notes on Bark and wood-boring beetles (Coleoptera: Bostrichidae; Curculionidae: Platypodinae and Scolytinae) of the Sweetgum (Liquidambar orientalis Mill.) Forest Nature Protection Area, with a new record for Turkish fauna. Journal of Food, Agriculture and Environment 11: 2178-2185, (2013).

[26] O. Sarıkaya and H. Sayın, Use of the red winged sticky traps for collecting bark and ambrosia beetles [Scolytinae (Coleoptera: Curculionidae)] on deciduous trees of Kasnak oak nature protection area in Isparta, Turkey. Research Journal of Biotechnology 11(9): 79-85, (2016).

[27] C. Tuncer, M. Knížek and J. Hulcr, Scolytinae in hazelnut orchards of Turkey: clarification of species and identification key (Coleoptera: Curculionidae). ZooKeys 710: 65-76, (2017).

[28] K.E. Schedl, Borkenkäfer aus der Türkei, III. Mitteilung 41:21-4, (1968).

[29] F.S. Bodenheimer, Türkiye'de Ziraata ve Ağaçlara Zararlı Olan Böcekler ve Bunlarla Savaş Hakkında Bir Etüd. Bayur Matbaası, Ankara, (1958). 